\title{
Current Safety Concerns with Human Papillomavirus Vaccine: A Cluster Analysis of Reports in VigiBase ${ }^{\circledR}$
}

\author{
Rebecca E. Chandler ${ }^{1} \cdot$ Kristina Juhlin $^{1} \cdot$ Jonas Fransson $^{1} \cdot$ Ola Caster $^{1,2}$ • \\ I. Ralph Edwards ${ }^{1}$ G. Niklas Norén ${ }^{1}$
}

Published online: 16 September 2016

(c) The Author(s) 2016. This article is published with open access at Springerlink.com

\begin{abstract}
Introduction A number of safety signals-complex regional pain syndrome (CRPS), postural orthostatic tachycardia syndrome (POTS), and chronic fatigue syndrome (CFS) have emerged with human papillomavirus (HPV) vaccines, which share a similar pattern of symptomatology. Previous signal evaluations and epidemiological studies have largely relied on traditional methodologies and signals have been considered individually.

Objective The aim of this study was to explore global reporting patterns for HPV vaccine for subgroups of reports with similar adverse event (AE) profiles.

Methods All individual case safety reports (reports) for HPV vaccines in VigiBase ${ }^{\circledR}$ until 1 January 2015 were identified. A statistical cluster analysis algorithm was used to identify natural groupings based on $\mathrm{AE}$ profiles in a data-driven exploratory analysis. Clinical assessment of the clusters was performed to identify clusters relevant to current safety concerns.

Results Overall, 54 clusters containing at least five reports were identified. The four largest clusters included $71 \%$ of the analysed HPV reports and described AEs included in the product label. Four smaller clusters were identified to include case reports relevant to ongoing safety concerns
\end{abstract}

Electronic supplementary material The online version of this article (doi:10.1007/s40264-016-0456-3) contains supplementary material, which is available to authorized users.

Rebecca E. Chandler

rebecca.chandler@who-umc.org

1 Uppsala Monitoring Centre, WHO Collaborating Centre for International Drug Monitoring, Uppsala, Sweden

2 Department of Computer and Systems Sciences, Stockholm University, Kista, Sweden (total of 694 cases). In all four of these clusters, the most commonly reported $\mathrm{AE}$ terms were headache and dizziness and fatigue or syncope; three of these four AE terms were reported in $>50 \%$ of the reports included in the clusters. These clusters had a higher proportion of serious cases compared with HPV reports overall (44-89\% in the clusters compared with $24 \%$ ). Furthermore, only a minority of reports included in these clusters included AE terms of diagnoses to explain these symptoms. Using proportional reporting ratios, the combination of headache and dizziness with either fatigue or syncope was found to be more commonly reported in HPV vaccine reports compared with non-HPV vaccine reports for females aged 9-25 years. This disproportionality remained when results were stratified by age and when those countries reporting the signals of CRPS (Japan) and POTS (Denmark) were excluded.

Conclusions Cluster analysis reveals additional reports of AEs following HPV vaccination that are serious in nature and describe symptoms that overlap those reported in cases from the recent safety signals (POTS, CRPS, and CFS), but which do not report explicit diagnoses. While the causal association between HPV vaccination and these AEs remains uncertain, more extensive analyses of spontaneous reports can better identify the relevant case series for thorough signal evaluation. 


\section{Key Points}

There are additional reports of adverse events (AEs) following HPV vaccination that are serious in nature and overlap with symptoms reported in cases from the recent safety signals of postural orthostatic tachycardia syndrome, complex regional pain syndrome, and chronic fatigue syndrome.

A causal association between HPV vaccination and these AEs remains uncertain.

Methods such as cluster analysis can help identify the relevant case series for thorough signal evaluation.

\section{Introduction}

Vaccines against the human papillomavirus (HPV) were initially introduced into public use 10 years ago, first with the 2006 European approval of the quadrivalent vaccine, Gardasil, and then subsequently with the 2007 approval of the bivalent vaccine, Cervarix. Since that time it has been estimated that 80 million girls and women worldwide have received the HPV vaccination [1].

Introduction of HPV vaccination into national vaccination programmes has been varied between countries, with Australia becoming the first country to introduce a government-funded National HPV Vaccination Program in 2007 [2]. Within the EU, Germany recommended vaccination as early as 2007, the UK implemented the HPV vaccination into the national vaccination programme in 2008, while the various Nordic countries incorporated the vaccination between 2009 and 2012 [3]. Although available for private purchase in the US since 2007, it was not until 2010 that HPV vaccines were required to be covered in insurance policies [4]. Japan introduced the vaccine in April 2010 and had uptake as high as $90 \%$ in those regions of the country in which the vaccine was available by government subsidy. By August 2014, 58 countries had introduced the HPV vaccine into their national immunisation programme for girls and also for boys in some countries [5].

In 2013, a number of safety signals arose for HPV vaccines: complex regional pain syndrome (CRPS) in Japan, postural orthostatic tachycardia syndrome (POTS) in Denmark, and long-lasting fatigue in the Netherlands [6-8]. Each of these diagnoses are generally poorly understood and largely under-recognised, particularly in the pediatric population, as revealed in the relative paucity of published data regarding their pathophysiology and epidemiology. The European Medicines Agency (EMA) reported a review of the safety concerns of POTS and CRPS in November 2015. The assessment primarily included a review of data submitted by the marketing authorisation holders (MAHs) and consultation with a scientific advisory group on vaccines. Although the EMA acknowledged the inherent difficulty in an evaluation of these signals ("Symptoms of CRPS and POTS may overlap with other conditions, making diagnosis difficult in both the general population and vaccinated individuals"), their final conclusion was that the current evidence does not suggest a causal association between HPV vaccines and POTS or CRPS [9]. In spite of these regulatory assurances, public concerns regarding the safety of HPV vaccines remain as revealed in the recent significant decrease in vaccine uptake in Denmark [10] and in the actions of patient advocacy groups collaborating globally [11].

The aim of this work was to explore VigiBase, the World Health Organization (WHO) international database of suspected adverse drug reactions, for additional relevant cases in the review of the serious safety signal for HPV vaccines which has been difficult to fully characterise by traditional signal detection methodology. We hypothesised that different reporters might use different $\mathrm{AE}$ terms to describe similar conditions, and yet there might be enough overlap between reports describing related conditions to identify subgroups of HPV vaccine reports with similar AE profiles. To achieve these aims, a novel data-driven approach to signal exploration was used: adverse event (AE) cluster analysis.

\section{Methods}

The data source for this investigation was VigiBase, the WHO international database of suspected adverse drug reactions. VigiBase is a computerised pharmacovigilance system in which information is recorded in a structured, hierarchical form. Reports are received from national pharmacovigilance centres in the 124 countries participating in the WHO Programme for International Drug Monitoring (PIDM). Reports submitted to national centres come from both regulated and voluntary sources. Limited details about each suspected adverse reaction are received by the Uppsala Monitoring Centre (UMC) [12].

All individual case safety reports (reports) for HPV vaccines received into VigiBase to 1 January 2015 were identified for use in this investigation. All analyses were performed on AE data at the Medical Dictionary for Regulatory Activities $\left(\right.$ MedDRA $^{\circledR}$ ) Preferred Term (PT)-level terminology. 
Seriousness designation is included in reports and is determined either by reporter or at local reporting, national pharmacovigilance centres. Commonly used standardisation of seriousness is as provided by International Conference on Harmonisation (ICH) Guidelines [13].

\subsection{Adverse Event Cluster Analysis}

Cluster analysis seeks to identify natural subgroups in data with closer resemblance between items within a subgroup than between items in different subgroups. It represents a form of unsupervised learning, where the algorithm is not provided with known examples from a number of prespecified classes or other information on the nature of the classes. Instead, the classes and their profiles are derived from data without additional guidance. Restricting our dataset to HPV case reports containing at least two AE terms, we applied latent class cluster analysis [14], which is based on a probabilistic mixture model in which each report is assumed to have been generated in a random process, drawn from one of a set of distinct latent classes, each with its overall relative frequency and set of probabilities for reported AE terms. To begin with, reports are randomly allocated to a latent class. A so-called expectation-maximisation (EM) iteration [15] is then employed by which the relative frequency and AE probabilities of each class are alternatingly re-estimated based on its allocated reports, and the reports are then re-allocated to latent classes based on the updated relative frequencies and $\mathrm{AE}$ profiles of these classes. In order to increase the stability of our overall solution, we applied so-called consensus clustering. EM iteration was thus repeated 100 times with random starting points, and final clusters derived as groups of reports that had been allocated to the same latent class as another report in the same cluster, in at least 90 of the 100 original solutions to the latent class cluster analysis.

\subsection{Clinical Review of Clusters}

All resultant clusters were reviewed independently by two different clinicians (RC and IRE) to determine the clinical scenario described by each cluster. Clusters with symptoms relevant for current safety concerns were selected for caselevel clinical review.

Cases within the identified clusters were reviewed for clinical coherence, with a focus on seriousness, which included review of seriousness criteria as well as clinical assessment of case narrative information, when available, for details regarding diagnostic procedures or results and impact on quality of life (such as quitting activities, missing school).

A case was deemed relevant to current safety concerns if the reported AEs, seriousness, and/or case narrative together would warrant inclusion of POTS, CRPS, or chronic fatigue syndrome (CFS) into a clinical differential diagnosis. Neither cases reporting alternate diagnoses as AE terms or including narrative information (risk factors, diagnostic results) suggestive of the potential for other diagnoses nor cases describing symptoms that had either resolved or had been ongoing for a short duration after vaccination (up to 1 week) were considered to be relevant.

\subsection{Proportional Reporting Ratios}

Upon identification of the combination of the AE terms headache and dizziness with either syncope or fatigue from the results of the cluster analysis, an investigation was made to explore if there was disproportionate reporting of this combination of AE terms in HPV vaccine reports compared with non-HPV vaccines in the same sex and age band (females 9-25 years). To match the time period in which HPV vaccines have been available, we limited the comparator group (non-HPV vaccine reports) to those received from 2005 onwards. Given that the age range described in the indication for HPV vaccines is somewhat wide, stratified analyses were also performed for the age groups 9-12 years, 13-16 years, 17-20 years, and 21-25 years, as a sensitivity analysis. Furthermore, given that the signals of CRPS and POTS arose from Japan and Denmark, respectively, adjusted proportional reporting ratios (PRRs) were calculated after exclusion of reports from these two countries. A further analysis was made to determine if there was disproportionality in the 'seriousness' of reports describing this combination of terms in HPV vaccine reports compared with non-HPV vaccines. PRRs were calculated [16].

\section{Results}

On 1 January 2015 there were a total of 10.3 million reports in VigiBase, and 39,953 HPV vaccine reports were entered into the clustering algorithm. The reports were sorted into a total of 4116 clusters with 54 of the clusters containing five or more reports. All 54 clusters are included in electronic supplementary Table 1.

The four largest clusters included $71 \%(28,502)$ of the analysed HPV vaccine reports and described AEs that are well characterised and are included in the product label, such as local and systemic events and vasovagal reactions. These clusters were further characterised by the fact that, on average, reports included only a few AE terms (three to four PTs) and were generally classified as non-serious (Fig. 1). Clinical review of the smaller clusters revealed that they described case series related to various clinical scenarios, such as cervical dysplasia (cluster 7), 

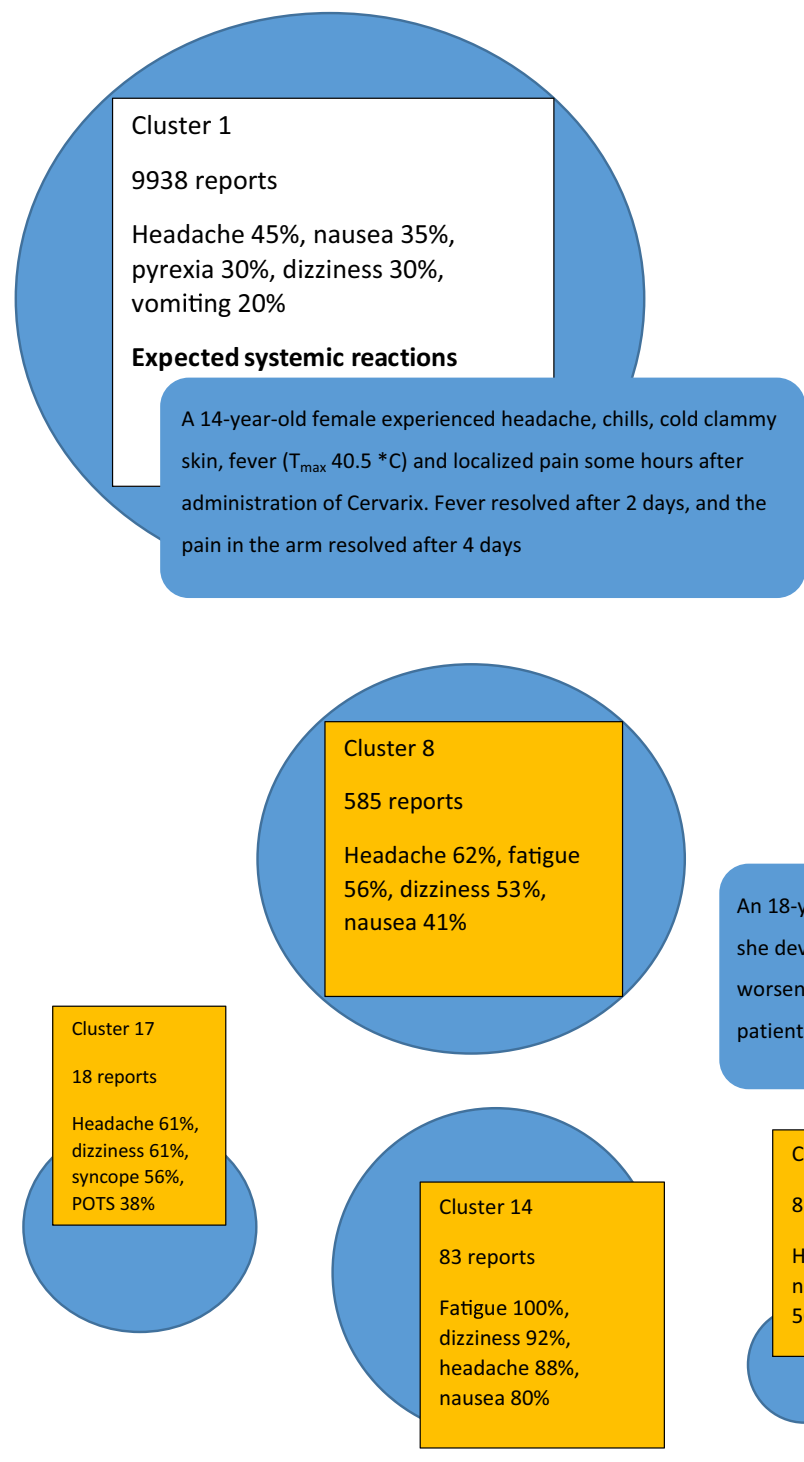

Cluster 2

7088 reports

Pruritis $17 \%$, urticaria $15 \%$, erythema $15 \%$, rash $15 \%$.

\section{Allergic/hypersensitivity reactions}

An 18-year-old female received her third dose of Gardasil. A few hours later, she developed an itchy rash all over her body. Two days later, the rash worsened and the patient also developed facial and tongue swelling. The

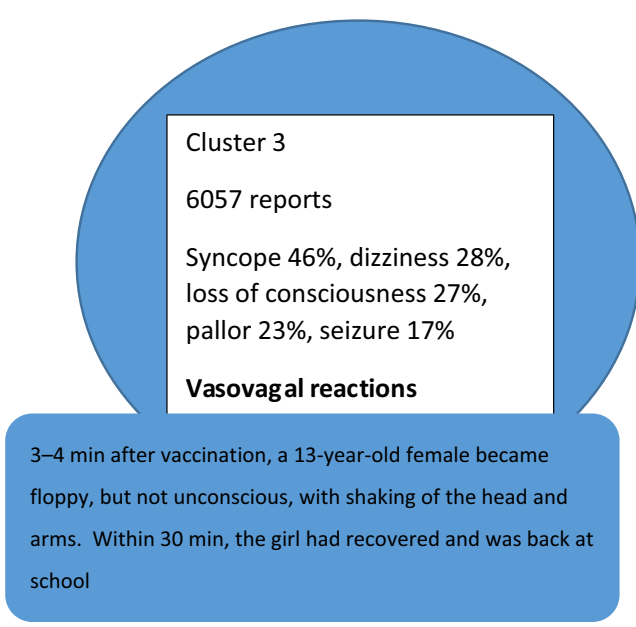

Fig. 1 Diagram of the four largest clusters and the four smaller clusters of interest. The four largest clusters described well charaterised clinical scenarios which are included in the product labels for HPV vaccines. Representative case narratives have been chosen to patient was treated with antihistamines and corticosteroids
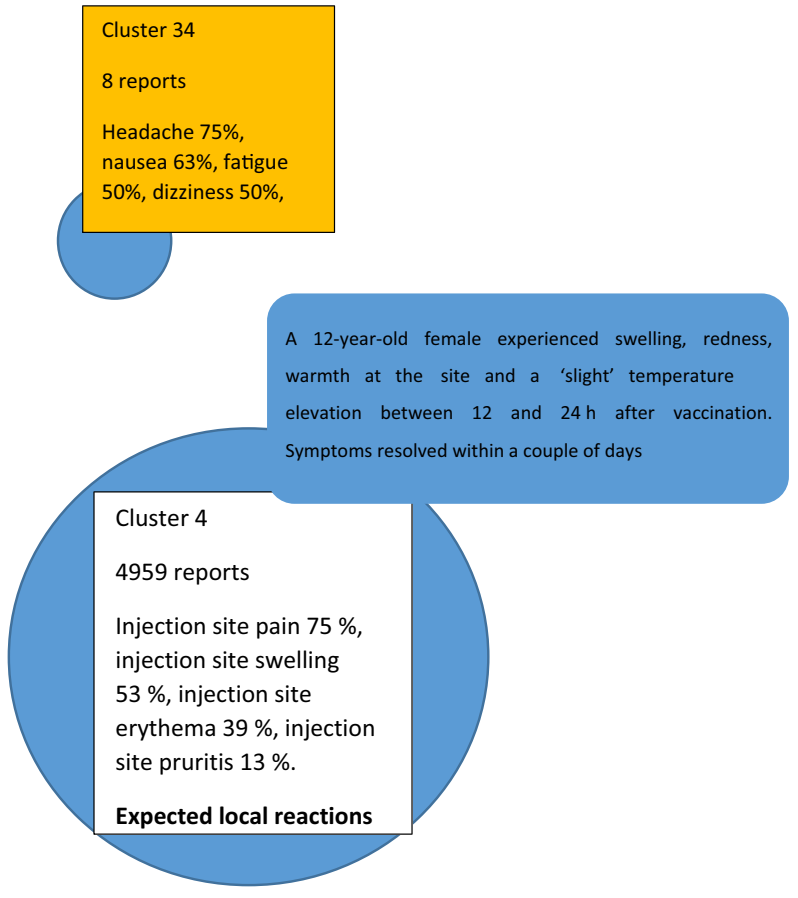

exemplify the types of cases included in these clusters. $T_{\max }$ Maximal recorded body temperature, POTS postural orthostatic tachycardia syndrome 
mononucleosis (cluster 21), and systemic lupus erythematosus (cluster 39) [electronic supplementary Table 1].

Four smaller clusters were identified as including case reports relevant to ongoing safety concerns. In all four of these clusters, the most commonly reported $\mathrm{AE}$ terms were headache and dizziness and fatigue or syncope; three of these four AE terms were reported in $>50 \%$ of the reports included in the clusters. These clusters had a higher proportion of serious cases compared with HPV reports overall (44-89\% in the clusters compared with $24 \%$ ). The most common criteria for a 'serious' designation was 'caused/ prolonged hospitalisation' ( $32 \%, 139 / 432$ serious reports), 'disabling/incapacitating' ( $27 \%, 111 / 432)$, or a combination of these two $(14 \%, 62 / 432)$.

Three of these clusters contained significant proportions of the total number of overall HPV reports contained within Vigibase reporting the AE terms of POTS (58\%, 61/106), CRPS $(20 \%, 15 / 76)$ and CFS (37 \%, 32/87); however, case reports including one of these diagnoses represented only a small proportion of the total cases included in the clusters (15\% of 694 reports) (Table 1)].

Individual-level review of cases within the clusters revealed further clinical coherence between the clusters reflected in the various patterns of less commonly reported AEs, including symptom terms (such as nausea, muscular weakness, disturbances in attention) and diagnostic procedure terms (such as electrocardiogram, magnetic resonance imaging head), as well as the impact on the life of the reporter, as identified from seriousness, reported AEs (such as activities of daily life impaired, quality of life decreased, bedridden, wheelchair user) and case narrative information. Case examples demonstrating an impact on the life of the reporter from each of the clusters include: "I [mother] am very frustrated and so is she. This has affected our entire family. I feel helpless and don't know how to help her" (cluster 8, case coded as 'non-serious'); "She was clever in school and at home, liked to go swimming, bicycling, dancing and so on. After the vaccinations with Gardasil she stopped being active, the life altered to the worst" (cluster 14, case coded as serious 'disabling/incapacitating', included both MedDRA terms 'quality of life decreased' and 'activities of daily living impaired'); "Missed lots of school due to NO energy and feeling bad. Will sometimes sleep 12 to 20 hours a day. Very healthy before the shot. She is tired of being sick" (cluster 17, case coded as 'non-serious'); "It's too late for my son whose life has been irrevocably altered/his life has never been the same" (cluster 34, case coded as 'non-serious', included the MedDRA term 'quality of life decreased').

Based on these individual-level clinical reviews, the majority of the total of 694 cases $(77.5 \%)$ included in these four clusters were deemed to be relevant to ongoing safety concerns.
A timeline of the years of receipt of the cases contained within the clusters into VigiBase reveals that cases have been received throughout the post-marketing life of HPV vaccines. The reporting countries represented in these clusters are the following: USA with 491 cases, Denmark with 88 cases, Japan with 39 cases, Germany with 28 cases, the United Kingdom with 23 cases, Ireland with 6 cases, Spain with 5 cases, France with 3 cases, Canada and Australia and Belgium and Norway with 2 cases, and Austria, Sweden, and Hungary with 1 case each. Those countries with earlier introduction of HPV vaccination into routine use reported the earliest cases (Australia, Germany the United States, and the UK) with an increasing contribution by those countries with a later introduction (Ireland, Japan, Denmark) (Fig. 2).

PRRs were calculated using 45,780 HPV vaccine reports and 32,839 non-HPV vaccine reports from females between the ages of 9 and 25 years. There were 596 $(1.3 \%) \mathrm{HPV}$ vaccine reports containing the AE combination of interest (headache and dizziness with either syncope or fatigue) compared with 175 reports $(0.53 \%)$ for other vaccines. The PRR was 2.44 (95\% confidence interval [CI] 2.07-2.89). The age-stratified analysis revealed that the PRR for this combination of AEs remained statistically significantly increased for HPV vaccines compared with other vaccines in all age groups, with the smallest value being 2.09 (95 \% CI 1.55-2.81). Additionally, the PRR for this combination of terms remained significant when reports from Japan and Denmark were excluded (2.28, $95 \%$ CI 1.92-2.70) [electronic supplementary Table 2].

When restricting analyses to only those reports with available information regarding 'seriousness', the reporting rate of serious reports with this combination of $\mathrm{AE}$ for HPV vaccines was $53.3 \%$ and $43.9 \%$ for other vaccines. The PRR is therefore calculated to be $1.21(1.00-1.47)$. In both the age-stratified analyses and the analysis excluding reports from Japan and Denmark, the PRR for serious reports with this combination of AEs were all $>1$, however these results were no longer statistically significant (electronic supplementary Table 3).

\section{Discussion}

Our analysis of global reports of suspected AEs following HPV vaccination has revealed a large number of reports with a pattern of AEs, including headache, dizziness, fatigue and syncope, distinguished from more common AEs by their serious nature (causing/prolonging hospitalisation and/or disabling/incapacitating), resulting in an impact on the quality of life of the patient. Included in this group are reports that have been labelled as POTS, CFS, and CRPS, but the majority of the reports lack explicit 
Table 1 Clusters identified as clinically relevant to evaluation of the safety concern, which has been variously identified as CRPS, POTS and CFS

\begin{tabular}{|c|c|c|c|c|}
\hline & Cluster 8 & Cluster 14 & Cluster 17 & Cluster 34 \\
\hline Total number of reports in the cluster & 585 & 83 & 18 & 8 \\
\hline \multirow[t]{5}{*}{ Top reported adverse events [MedDRA] $(\%)$} & Headache (62) & Fatigue (100) & Headache (61) & Headache (75) \\
\hline & Fatigue (56) & Dizziness (92) & Dizziness (61) & Nausea (63) \\
\hline & Dizziness (53) & Headache (88) & Syncope (56) & Fatigue (50) \\
\hline & Nausea (41) & Nausea (80) & POTS (39) & Dizziness (50) \\
\hline & Arthralgia (31) & Arthralgia (71) & EEG normal (33) & Arthralgia (38) \\
\hline \multirow[t]{8}{*}{ Other adverse events of interest (\%) } & Syncope (22) & Syncope (25) & Fatigue (33) & Syncope (13) \\
\hline & $\begin{array}{l}\text { Upper abdominal } \\
\text { pain }(16)\end{array}$ & $\begin{array}{l}\text { Abdominal pain } \\
\text { (37) }\end{array}$ & $\begin{array}{l}\text { Abdominal pain } \\
\text { (11) }\end{array}$ & $\begin{array}{l}\text { Abdominal pain } \\
\text { (13) }\end{array}$ \\
\hline & $\begin{array}{l}\text { Muscular } \\
\text { weakness (15) }\end{array}$ & $\begin{array}{l}\text { Muscular } \\
\text { weakness (48) }\end{array}$ & $\begin{array}{l}\text { Muscular } \\
\quad \text { weakness (11) }\end{array}$ & $\begin{array}{l}\text { Abdominal pain } \\
\text { upper (13) }\end{array}$ \\
\hline & $\begin{array}{l}\text { Disturbance in } \\
\text { attention (14) }\end{array}$ & $\begin{array}{l}\text { Disturbance in } \\
\text { attention }(66)\end{array}$ & $\begin{array}{l}\text { Loss of } \\
\text { consciousness }\end{array}$ & $\begin{array}{l}\text { Muscular } \\
\text { weakness (13) }\end{array}$ \\
\hline & Palpitations (13) & Palpitations (39) & $(28)$ & Disturbance in \\
\hline & Pain $(21)$ & Pain (46) & Palpitations (22) & attention (38) \\
\hline & & & Pain (6) & Palpitations (38) \\
\hline & & & & Pain (13) \\
\hline \multirow[t]{5}{*}{ Origin of reports $(\%)$} & USA (75) & USA (40) & USA (89) & USA (50) \\
\hline & Denmark (9) & Denmark (39) & Germany (6) & Denmark (25) \\
\hline & Japan (6) & Japan (5) & Spain (6) & Germany (13) \\
\hline & Germany (4) & UK (5) & & Japan (13) \\
\hline & UK (3) & Germany (5) & & \\
\hline Mean number of reported MedDRA terms per case report & 14.6 & 41.7 & 12.9 & 8.5 \\
\hline \multirow[t]{5}{*}{ Reports containing certain diagnosis-related terms (\%) } & POTS (6) & POTS (23) & POTS (39) & 0 \\
\hline & CFS (4) & CFS (11) & & \\
\hline & PVFS (2) & PVFS (7) & & \\
\hline & CRPS (2) & CRPS (4) & & \\
\hline & FM (2) & FM (5) & & \\
\hline Percentage of 'serious' reports & $58 \%$ (343 reports) & $89 \%$ (74 reports) & $44 \%$ (8 reports) & $88 \%$ (7 reports) \\
\hline \multirow[t]{4}{*}{ Reports containing diagnostic testing-related terms (\%) } & CT (16) & CT (11) & CT (33) & 0 \\
\hline & MRI (22) & MRI (11) & MRI (39) & \\
\hline & ECG (14) & ECG (3.6) & ECG (44) & \\
\hline & EEG (7.9) & EEG (2.4) & EEG (50) & \\
\hline $\begin{array}{l}\text { Percentage of reports containing MedDRA code } \\
\text { 'Activities of daily living impaired' }\end{array}$ & $20 \%(117 / 585)$ & $19 \%(16 / 83)$ & $5.5 \%(1 / 18)$ & $1 / 8$ (serious case) \\
\hline $\begin{array}{l}\text { Percentage of reports containing MedDRA code 'Quality } \\
\text { of life decreased' }\end{array}$ & $1.4 \%(8 / 585)$ & $4.8 \%(4 / 83)$ & 0 & $\begin{array}{l}1 / 8 \text { (non-serious } \\
\text { case) }\end{array}$ \\
\hline $\begin{array}{l}\text { Percentage of serious reports with designation of } \\
\text { 'disabling/incapacitating' }\end{array}$ & $47 \%(162 / 343)$ & $57 \%(42 / 74)$ & 0 & $1 / 7$ \\
\hline $\begin{array}{l}\text { Percentage of cases determined to be clinically relevant to } \\
\text { ongoing safety concerns }\end{array}$ & $75 \%(438 / 585)$ & $95 \%(79 / 83)$ & $72 \%(13 / 18)$ & $100 \%(8 / 8)$ \\
\hline
\end{tabular}

CRPS complex regional pain syndrome, POTS postural orthostatic tachycardia syndrome, $C F S$ chronic fatigue syndrome, MedDRA Medical Dictionary for Regulatory Activities, $C T$ any MedDRA term containing 'computerized tomography', MRI any MedDRA term containing 'nuclear magnetic resonance imaging', $E C G$ any MedDRA term containing 'electrocardiogram', EEG any MedDRA term containing 'electroencephalogram', $P V F S$ post viral fatigue syndrome, $F M$ fibromyalgia

diagnoses. Furthermore, our analyses suggest that the combination of headache and dizziness with either fatigue or syncope is reported significantly more often in HPV vaccine reports compared with non-HPV vaccine reports for females aged 9-25 years; this disproportionality remains when results are stratified by age and when those countries reporting the signals of CRPS (Japan) and POTS (Denmark) are excluded. 


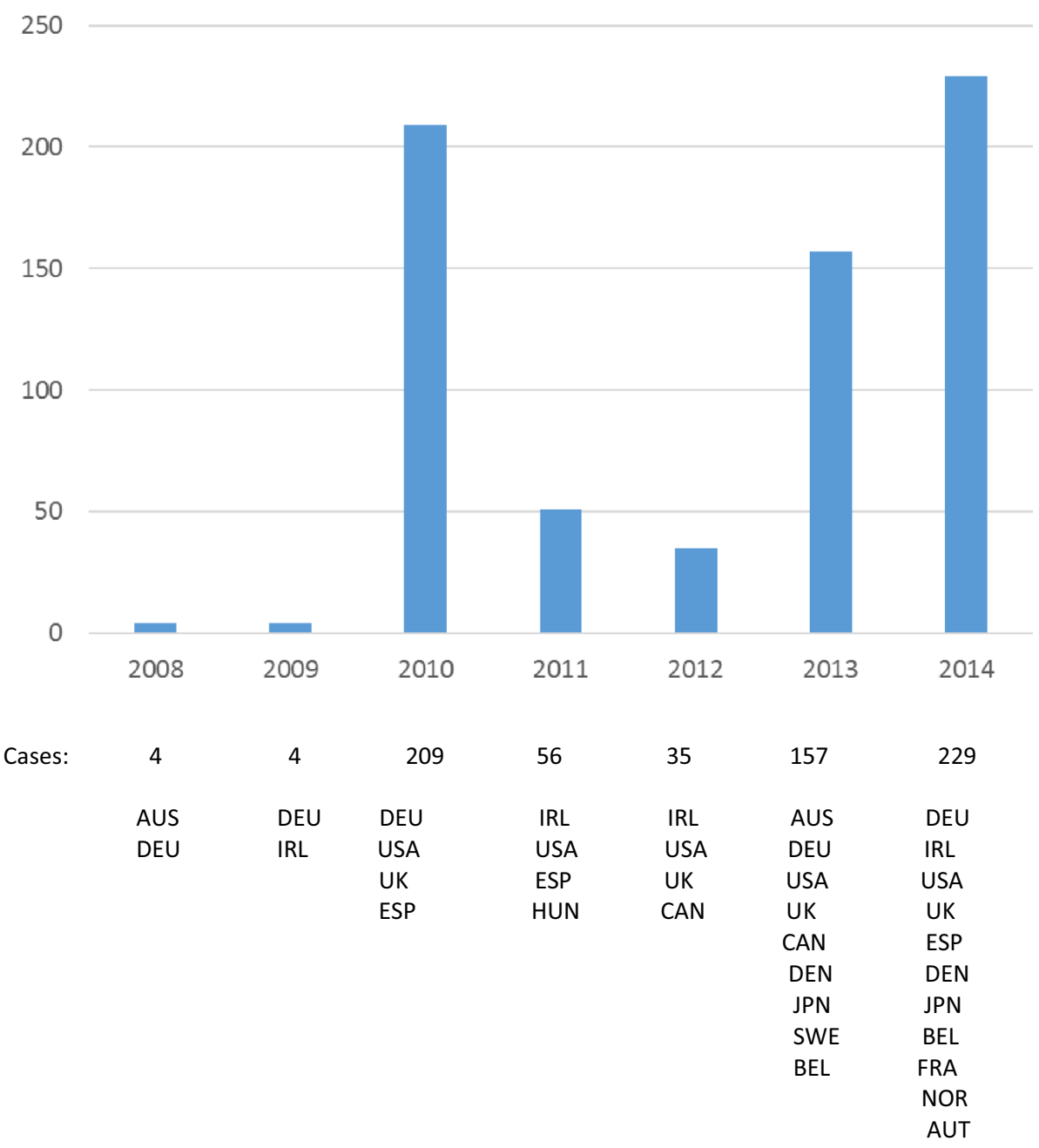

Fig. 2 Timeline displaying the number of case reports (per year and by country of origin) that were included in the four clusters. As can be seen from the figure, case reports included in the clusters have been received throughout the whole of the HPV vaccine postmarketing period, with the earliest reports received from those countries who were the first to introduce the HPV vaccine into their vaccination programmes. Countries of origin are diverse, with the first reports from Denmark and Japan (signalling countries) received in 2013. In

Given concerns regarding the influence in reporting secondary to media, we restricted our analyses to reports that had been collected up to 1 January 2015 in order to reduce any influence in reporting secondary to both the widely publicised regulatory action of the EMA taken in July 2015 and the widely viewed television program 'De vaccinerede piger', which first aired in Denmark in March 2015 and was subsequently placed on YouTube with English subtitles. In addition, we have shown that cases within the clusters have been distributed over the entire postmarketing life of HPV vaccines, with cases first reported from those countries that were the first to recommend and/or implement HPV vaccination into routine use. Furthermore, in our PRR analyses, we have found an that same year, the largest number of reports were received from the USA (64) Denmark (55) the UK (13), and Germany (12). In 2010, a large backlog of vaccine reports from the USA, which were not specific to HPV vaccines, were entered into VigiBase. HPV human papillomavirus, AUS Australia, DEU Germany, IRL Ireland, USA United States of America, UK United Kingdom, ESP Spain, HUN Hungary, CAN Canada, DEN Denmark, JPN Japan, SWE Sweden, BEL Belgium, FRA France, NOR Norway, AUT Austria

increased reporting of this combination of $\mathrm{AE}$ events in HPV vaccines compared with other vaccines, even when reports from Japan and Denmark are excluded. Finally, the proportion of serious cases among reports with the $\mathrm{AE}$ combination was at least as high (but not significantly higher) for HPV vaccines as for non-HPV vaccines, which might not have been so if attention in the media would have led to increased reporting, primarily of less severe events.

The greatest limitation of this study is the lack of information on many of the reports in VigiBase. Missing data means that it may be difficult to make a clinical judgment regarding case relevancy, and thus there is the potential for a lack of consistency between different 
assessors. Consequently, there may be a lower or greater number of relevant cases within the clusters that would contribute to the final signal. Given the high proportion of cases considered relevant from our review $(77.5 \%)$, some variation in clinical judgment between assessors is unlikely to change the overall conclusion of our analysis.

The cases within the clusters are notably similar to those described in a number of safety signals for the HPV vaccines. In The Netherlands, the Pharmacovigilance Centre Lareb has published the document "Long-lasting adverse events following immunization with Cervarix ${ }^{\circledR}$ ", [8]. Given a large number of reports of long lasting ( $>2$ months) AEs following immunisation, a survey to enhance the clinical documentation level of these reports was performed. Overall 230 reports were considered relevant. Several combinations of frequently reported AEs were found, with the most common being fatigue, headache and musculoskeletal discomfort. Furthermore, the follow-up survey revealed that these long-lasting, unexplained symptoms have had a considerable impact on both the lives of the girls and their family members. In Japan, Kinoshita et al. reported a cohort of 40 subjects in whom they described headaches, general fatigue, coldness of the legs, limb pain and weakness. Eighteen girls met the criteria for a diagnosis of CRPS and, using the Schellong test, four were identified as having POTS. The authors in Japan hypothesised that the symptoms could be explained by abnormal peripheral sympathetic responses [6]. In Denmark, Brinth et al. reported a cohort of 35 subjects in whom they described nausea, chronic headache, fatigue, palpitations, reduced cognitive function, skin changes, intermittent tremor/myoclonic twitches, neuropathic pain, sleep disturbances, and muscular weakness. All subjects were diagnosed with orthostatic intolerance [7]. In the US, Blitshteyn reported a case series whose symptoms included dizziness, fatigue, nausea, weight loss and exercise intolerance [17], while in Mexico, Martinez-Lavín described two patients with fibromyalgia-type symptoms, including severe pain, insomnia, profound fatigue, and paraesthesia [18]. A signal of gastrointestinal motility disorders from the UMC described a case series of 26 subjects, in whom 15 reported concomitant fatigue, 13 reported either headache or migraine, and 9 reported dizziness [19]. The most recent publication is a report from Italy that describes a case series of 18 girls (aged 12-24 years) who were referred to a 'Second Opinion Medical Network' for the evaluation of 'neuropathy with autonomic dysfunction' after HPV vaccination; all girls complained of long-lasting and invalidating somatoform symptoms (including asthenia, headache, cognitive dysfunctions, myalgia, sinus tachycardia and skin rashes) [20]. It is acknowledged that these publications represent case series and thus have the limitations that are traditionally associated with these types of reports. However, the collection of a number of case series displaying similar patterns of AEs from geographically distributed locations potentially adds to the overall strength of the signal.

The EMA referral procedure focused on the two specific syndromes of POTS and CRPS; the reviews of the safety database and observed versus expected analyses performed on spontaneous reports by the MAHs considered each of these two syndromes separately. Furthermore, although it was requested that the MAH use 'common search strategies' to identify potentially undiagnosed case reports with combinations of signs and symptoms common in CRPS or POTS, the details of these search strategies have not been provided in the publically available assessment report. However, the total number of additional cases identified by these queries, even prior to their assessment according to CRPS and POTS diagnostic criteria, were less than the cases identified by the explicit reporting of the terms POTS and CRPS [9]. In contrast, our analysis identified a greater number of potentially undiagnosed cases than the total number of cases that had been labelled with one of these diagnoses.

There have been a number of epidemiological investigations regarding safety concerns of the HPV vaccine that have relied primarily on the reporting of a specific diagnosis or single-symptom concept, either as a diagnostic billing code or single or synonymous AE terms to define the relevant case series. An epidemiological study in The Netherlands was designed to investigate the potential for an increased risk of migraine headaches after HPV vaccination [21]. As part of a proactive pharmacovigilance plan, the Medicines and Health Regulatory Authority (MHRA) performed an epidemiological study using self-controlled case-series methodology in which they identified cases reporting 'fatigue-containing' terms, such as chronic fatigue, chronic fatigue syndrome, post-viral fatigue and fibromyalgia [22]. Although laudable that it considered a variety of fatigue-related terms, the study did not account for co-reported symptoms of a more varied nature. Similarly, while the results of the largest post-authorisation safety studies have been reassuring overall, it must be remembered that such studies have used predefined, welldefined medical pathologies as endpoints [23-26]. The use of epidemiological studies that require specific case definitions are useful to quantify the risks of safety concerns after a signal has been identified and well-characterised from AE reports.

Given the lack of consensus on the specific diagnosis these spontaneous reports describe, a focus on symptomatology and seriousness in combination with an investigation of the underlying pathology may be required to fully elucidate this safety signal. Evidence of a common pathophysiology in girls with this symptomatology is, in 
fact, currently emerging, with a focus on autoantibodies to $\mathrm{G}$ protein-coupled receptors in the nervous system, such as $\beta 2$-adrenergic and muscarinic- 2 receptors which have been isolated from one subject in the US [27] and in a large proportion of a sample of patients in Denmark (Mehlsen J, personal communication). These results could explain the variety of labels that have been used to explain this symptomatology, as autoantibodies to these receptors have been previously linked with CRPS, POTS, and CFS [28-31].

A causal association with the HPV vaccine remains uncertain; however, we believe that a more thorough investigation of this signal is required to ensure continued public trust in both vaccination programmes and regulatory authorities.

\section{Conclusions}

Cluster analysis reveals additional reports of AEs following HPV vaccination, which are serious in nature and with overlap in signs and symptoms to recent safety signals for POTS, CRPS, and CFS, but without explicit diagnoses. Furthermore, there is the suggestion of disproportional reporting of this pattern of symptomatology in reports after HPV vaccination compared with other vaccines in females 9-25 years of age. This disproportionality remained even when those countries reporting the signals of CRPS (Japan) and POTS (Denmark) were excluded.

A causal association between these AEs and HPV vaccination remains uncertain; however, given the medical seriousness of this safety concern, we believe that a more definitive study of the findings presented here is essential to ensure continued confidence in the HPV vaccine.

Our study illustrates how more extensive analyses of spontaneous reports can better identify the relevant case series for thorough signal evaluation.

Acknowledgments The authors are indebted to the national centres who make up the WHO PIDM and contribute reports to VigiBase. However, the opinions and conclusions are not necessarily those of the various centres nor of the WHO.

\section{Compliance with Ethical Standards}

Funding This work was entirely funded by the UMC, WHO Collaborating Centre for International Drug Monitoring.

Conflict of interest Rebecca Chandler, Kristina Juhlin, Jonas Fransson, Ola Caster, I. Ralph Edwards and G. Niklas Noren have no conflicts of interest that are directly relevant to the content of this study.

Open Access This article is distributed under the terms of the Creative Commons Attribution-NonCommercial 4.0 International License (http://creativecommons.org/licenses/by-nc/4.0/), which permits any noncommercial use, distribution, and reproduction in any medium, provided you give appropriate credit to the original author(s) and the source, provide a link to the Creative Commons license, and indicate if changes were made.

\section{References}

1. HPV vaccines: EMA confirms evidence does not support that they cause CRPS or POTS. EMA/749763/2015. 2015. Available at: http://www.ema.europa.eu/docs/en_GB/document_library/ Referrals_document/HPV_vaccines_20/Opinion_provided_by_ Committee_for_Medicinal_Products_for_Human_Use/ WC500196773.pdf.

2. Tabrizi S, Brotherton J, Kaldor J, Skinner SR, Cummins E, Lui E, et al. Fall in human papillomavirus prevalence following a national vaccination program. $J$ Infect Dis. 2012;206(11):1645-51.

3. European Centre for Disease Prevention and Control. ECDC guidance. Introduction of HPV vaccines in European Union countries: an update. 2012. Available at: http://ecdc.europa.eu/en/ publications/Publications/20120905_GUI_HPV_vaccine_update. pdf.

4. ACA Preventative Services Benefits for Women and Pregnant Women. Immunization for women. Retrieved 10 Dec 2015.

5. Human papillomavirus vaccines: WHO position paper, October 2014. Wkly Epidemiol Rec. 2014;89(43):465-491. http://www. ncbi.nlm.nih.gov/pubmed/25346960.

6. Kinoshita T, Abe R-T, Hineno A, Tsunekawa K, Nakane S, Ikeda S. Peripheral sympathetic nerve dysfunction in adolescent Japanese girls following immunization with the human papillomavirus vaccine. Intern Med. 2014;53:2185-200.

7. Brinth LS, Pors K, Theibel AC, Mehlsen J. Orthostatic intolerance and postural tachycardia syndrome as suspected adverse effects of vaccination against human papilloma virus. Vaccine. 2015;33(22):2602-5.

8. Lareb. Long-lasting adverse events following immunization with Cervarix $^{\circledR}$. 2015. Available at: http://databankws.lareb.nl/ Downloads/Lareb_rapport_HPV_dec15_03.pdf.

9. European Medicines Agency assessment report. Review under Article 20 of Regulation (EC) No 726/200411. 2015. Available at: http://www.ema.europa.eu/docs/en_GB/document_library/ Referrals_document/HPV_vaccines_20/Opinion_provided_by_ Committee_for_Medicinal_Products_for_Human_Use/WC50019 7129.pdf.

10. Statens Serum Institut. Available at: http://www.ssi.dk/ Smitteberedskab/Sygdomsovervaagning/VaccinationSurveillance. aspx ?vaccination $=5 \&$ xaxis $=$ Cohort $\&$ se $=0 \&$ landsdel $=100 \&$ show $=$ Graph\&datatype $=$ Vaccination\&extendedfilters $=$ True\#HeaderText. Accessed 3 May 2016.

11. Sanevax. Open letter to EMA re: HPV vaccine safety. 2016 Available at: http://sanevax.org/open-letter-ema-hpv-vaccinesafety/.

12. Lindquist M. Vigibase, the WHO Global ICSR Database System: basic facts. Drug Inf J. 2008;42:409-19.

13. ICH Harmonised Tripartite Guideline. Maintenance of the ICH guideline on clinical safety data management: data elements for transmission of individual case safety reports E2B (R2). Available at: http://www.ich.org/fileadmin/Public_Web_Site/ICH_Products/ Guidelines/Efficacy/E2B/Step4/E2B_R2_Guideline.pdf.

14. Vermunt JK, Magidson J. Latent class cluster analysis. In: Hagenaars J, McCutcheon A, editors. Applied latent class analysis. Cambridge: Cambridge University Press; 2002. p. 89-106. 
15. Dempster AP, Laird NM, Rubin DB. Maximum likelihood from incomplete data via the EM algorithm. J R Stat Soc Series B Stat Methodol. 1977;39(1):1-38.

16. Evans SJW, Waller PC, Davis S. Use of proportional reporting ratios (PRRs) for signal generation from spontaneous adverse drug reaction reports. Pharmacoepidemiol Drug Saf. 2001;10(6):483-6.

17. Blitshteyn S. Postural tachycardia syndrome following human papilloma vaccination. Eur J Neurol. 2014;21(1):135-9.

18. Martínez-Lavín M. Fibromyalgia-like illness in 2 girls after human papillomavirus vaccination. $\mathrm{J}$ Clin Rheumatol. 2014;20(7):392-3.

19. Chandler R, Hult S, Caduff-Janosa P. Human papilloma virus vaccines and gastrointestinal motility disorders. Drug Saf. 2015;38(10):976.

20. Palmieri B, Poddighe D, Vadalà M, Laurino C, Carnovale C, Clementi E. Severe somatoform and dysautonomic syndromes after HPV vaccination: case series and review of literature. Immunol Res. 2016. doi:10.1007/s12026-016-8820-z.

21. Van't Klooster, de Ridder MAJ, Kemmerem JM, van der Lei J, Dekker F, Sturkenboom M, et al. Examining a possible association between human papilloma virus (HPV) vaccination and migraine: results of a cohort study in the Netherlands. Eur $\mathbf{J}$ Pediatr. 2015;174:641-649.

22. Donegan K, Beau-Lejdstrom R, King B, Seabroke S, Thomson A, Bryan P. Bivalent human papillomavirus vaccine and the risk of fatigue syndromes in girls in the UK. Vaccine. 2013;31(43):4961-7.

23. Arnheim-Dahlstrom L, Pasternak B, Svanstrom H, Sparen P, Hviid A. Autoimmune, neurological, and venous thromboembolic adverse events after immunisation of adolescent girls with quadrivalent human papillomavirus vaccine in Denmark and Sweden: cohort study. BMJ. 2013;347:f5906.
24. Klein NP, Hansen J, Chao C, Velicer C, Emery M, Slezak J, et al. Safety of quadrivalent human papillomavirus vaccine administered routinely to females. Arch Pediatr Adolesc Med. 2012;166(12):1140-8.

25. Grönlund O, Herweijer E, Sundström K, Arnheim-Dahlström L. Incidence of new-onset autoimmune disease in girls and women with pre-existing autoimmune disease after quadrivalent human papillomavirus vaccination: a cohort study. J Intern Med. 2016. doi:10.1111/joim.12535.

26. Willame C, Rosillon D, Zima J, Angelo MG, Stuurman AL, Vroling H, et al. Risk of new onset autoimmune disease in 9- to 25-year-old women exposed to human papillomavirus-16/18 AS04-adjuvanted vaccine in the United Kingdom. Hum Vaccin Immunother. 2016. doi:10.1080/21645515.2016.1199308.

27. Hendrickson JE. Human papilloma virus vaccination and dysautonomnia: considerations for autoantibody testing and HLA typing [letter]. Vaccine. 2016;34(38):4468.

28. Kohr D, Singh P, Tschernatsch M, Kaps M, Pouokam E, Diener $M$, et al. Autoimmunity against the $\beta 2$ adrenergic receptor and muscarinic-2 receptor in complex regional pain syndrome. Pain. 2011;152(12):2690-700.

29. Li H, Yu X, Liles C, Khan M, Vanderlinde-Wood M, Galloway A, et al. Autoimmune basis for postural tachycardia syndrome. J Am Heart Assoc. 2014;3(1):e000755.

30. Dubey D, Hopkins S, Vernino S. M1 and M2 muscarinic receptor antibodies among patients with Postural Orthostatic Tachycardia Syndrome: potential disease biomarker [abstract]. Available at: http://www.dysautonomiainternational.org/pdf/ Vernino_Muscarinic_Abstract.pdf.

31. Loebel M, Grabowski P, Heidecke H, Bauer S, Hanitsch LG, Wittke $\mathrm{K}$, et al. Antibodies to $\beta$ adrenergic and muscarinic cholinergic receptors in patients with chronic fatigue syndrome. Brain Behav Immun. 2016;52:32-9. 